\title{
Heating and dynamics of the quiet solar chromosphere
}

\author{
Wolfgang Kalkofen \\ Harvard-Smithsonian Center for Astrophysics \\ email: wkalkofen@cfa.harvard.edu
}

\begin{abstract}
The quiet solar chromosphere in regions with negligible magnetic field is believed to be heated by acoustic waves. But their energy flux, measured in the upper photosphere with the Transition Region And Coronal Explorer (TRACE), has been found to be insufficient to account for the radiative emission from the chromosphere. Wedemeyer-Böhm et al. (2007) and Cuntz et al. (2007), employing a 3D hydrodynamical model by Wedemeyer et al. (2004), have proposed that the spatial resolution of TRACE is inadequate to resolve intensity fluctuations that occur on small spatial scales. This paper accepts the principle of spatial averaging by TRACE as a qualitative explanation for the low acoustic flux but finds that the hydrodynamical model is too much simplified in the treatment of radiative energy exchange to provide a quantitative measure of the suppression of the fluctuations. The heating mechanism of the chromosphere thus remains an open question.
\end{abstract}

Keywords. waves, hydrodynamics, radiative transfer, Sun: atmosphere, Sun: photosphere, Sun: chromosphere

\section{Introduction}

The theory of chromospheric heating and structure has never been put on a firm observational basis. While it is generally believed that the dark interior of supergranulation cells, and therefore most of the quiet chromosphere, is heated by the dissipation of acoustic waves, all attempts to measure the acoustic flux via observed intensity fluctuations of radiation originating in the photosphere have failed to detect sufficient wave energy for balancing the radiative cooling of the chromosphere. A recent measurement using the Transition Region and Coronal Explorer (TRACE) for observations from space fared no better (Fossum \& Carlsson 2005a, 2005b, 2006), yielding less than $10 \%$ of the flux they expected, actually less than $4 \%$ of the flux they should have expected. More recent observations using Hinode (Carlsson et al. 2007), with $6 \%$ of the required flux detected in acoustic waves, improved the agreement between predicted and observed fluxes only marginally.

Two explanations were offered to account for the shortfall in acoustic flux: One, by Fossum \& Carlsson (2005b), suggested that the heating of the chromosphere is not by acoustic waves but by processes related to magnetic fields; and the other, by Wedemeyer-Böhm et al. (2007) and Cuntz et al. (2007), suggested that the spatial resolution of TRACE does not allow the small-scale velocity fluctuations responsible for acoustic heating to be detected.

The explanation for the missing acoustic flux in terms of waves related to the magnetic field was addressed by Kalkofen (2007) who showed that the spectroscopic features of radiation emerging from the chromosphere lacked the known indicators of transverse (kink) waves and instead showed only the indicators of acoustic waves, implying that the chromosphere is heated by acoustic waves. At the IAU symposium on Isla de Margarita, a 
further explanation of chromospheric heating without involving magnetic fields appeared plausible: in terms of internal gravity waves with significant energy flux in the photosphere, discussed by T. Straus. But the properties of internal gravity waves may make them unlikely heating agents. They are excluded from the purely vertical direction and would therefore depend on propagation at an oblique angle relative to the vertical. This would make it difficult for the wave energy to reach higher layers of the chromosphere, although internal gravity waves may play a role even in coronal loops (Mendoza-Briceño \& Erdélyi 2006). It is worth noting that impulsive excitation of acoustic waves generates also internal gravity waves (Bodo et al. 2000), so there is potentially high energy flux in these waves. But heating by internal gravity waves is a largely unexplored area.

Observations of $\mathrm{K}_{2 \mathrm{v}}$ bright points by Liu (1974) and simulations of $\mathrm{H}_{2 \mathrm{v}}$ bright points by Carlsson \& Stein (1995) link Ca II bright points in the chromosphere to velocity fluctuations in the photosphere immediately below, a behavior that is implausible for internal gravity waves, which travel preferentially in the horizontal direction. An additional difficulty is that the $5 \mathrm{mHz}$ wave frequency, as the Brunt-Väisälä cutoff frequency, is the upper limit for frequencies in the propagating range of internal gravity waves; but as the acoustic cutoff frequency, is the lower limit for propagating acoustic waves; the latter appear to be more in accord with the power spectrum in the so-called nonmagnetic medium, observed by Lites et al. (1993), but the case in favor of acoustic waves is not clear cut.

The most plausible mechanism of chromospheric heating in the internetwork (nonmagnetic) chromosphere would appear to be dissipation by acoustic waves. The explanation of the low observed acoustic flux in terms of the spatial resolution of TRACE is the topic of this paper. We ask whether three-dimensional hydrodynamic simulations have reached a level of maturity where they can be used for quantitative answers or are only able to provide qualitative estimates. In Section 2 we discuss the height-integrated radiative cooling rate of the nonmagnetic chromosphere, in Section 3 we describe temperature fluctuations in the hydrodynamical model of Wedemeyer et al. (2004: hereafter W04), in Section 4 we investigate a likely reason for the large temperature fluctuations predicted by the hydrodynamical model, and in Section 5 we summarize the findings.

\section{The radiative cooling rate}

The empirical temperature structure of the average model of Vernazza et al. (1981: hereafter VAL) was determined by matching the average observed emergent intensity to the intensity predicted from the empirical temperature in important spectroscopic features, mainly the $\mathrm{H}$ and $\mathrm{K}$ lines and the infrared triplet of $\mathrm{Ca}$ II, the $\mathrm{h}$ and $\mathrm{k}$ lines of $\mathrm{Mg} \mathrm{II}$ and the bound-free and free transitions in $\mathrm{H}^{-}$(see Fig. 49 of VAL). The model temperature was adjusted so as to optimize the fit of the two sets of intensities. The heightintegrated cooling rate of the average empirical model, called VAL-C, is $4.3 \mathrm{~kW} / \mathrm{m}^{2}$.

Most of the opacity sources providing the cooling of the atmosphere were not required for establishing the temperature structure and therefore were not included in the model; they were added by Anderson \& Athay (1989), mainly lines of Fe II, for which microturbulent broadening was assumed. This more complete model had the larger cooling rate of $14 \mathrm{~kW} / \mathrm{m}^{2}$ (see Table 1). The major uncertainty of this model is the absence of macroscopic velocity fields. But even with the simplification of leaving out organized flows, the resulting cooling rate is probably closer to the real solar situation than is that of the wave models of Carlsson \& Stein (1995) and W04, which exhibit much more dynamical behavior than is seen in solar observations of the quiet chromosphere. 


$\begin{array}{lcc}\text { Table } 1 & \text { Cooling rate of the nonmagnetic chromosphere } \\ \text { Reference } & \text { Power }\left[\mathrm{kW} / \mathrm{m}^{2}\right] & \text { relative power } \\ \text { Anderson \& Athay (1989) } & 14 & 1.0 \\ \text { VAL-C } & 4.3 & 0.3 \\ \text { Fossum \& Carlsson (2006) } & 0.51 & 0.04 \\ \text { Carlsson et al. }(2007) & 0.80 & 0.06\end{array}$

The $14 \mathrm{~kW} / \mathrm{m}^{2}$ empirical cooling rate is the target flux to be found in observations. But the observations with TRACE by Fossum and Carlsson (2006) found only $510 \mathrm{~W} / \mathrm{m}^{2}$ (with a correction by Carlsson et al. 2007), which is less than $4 \%$ of the rate implied by observations of the emergent radiation from the quiet internetwork chromosphere. A more recent space measurement, with Hinode (Carlsson et al. 2007), found $800 \mathrm{~W} / \mathrm{m}^{2}$, which is $6 \%$ of the target flux and thus still insufficient to heat the nonmagnetic chromosphere.

\section{Temperature fluctuations in the hydrodynamical model}

The hydrodynamical model of W04 that is employed to explain the low acoustic flux observed with TRACE is a 3D wave model in which acoustic waves are generated from the turbulent flow of the convection without additional assumptions. The most important simplification in the physics of the problem is the neglect of almost all opacity sources responsible for the radiative exchange of energy between hot and cool regions. The question to be posed is whether observations can help in sorting out the realism of the dynamical features of the model, and in particular, whether the low temperatures during cool phases and the large amplitude of the temperature fluctuations agree qualitatively with observations of the Sun.

Figure 1, from W04, shows four simultaneous horizontal cuts through the chromosphere, giving the temperature structure at the heights of $500 \mathrm{~km}$, i.e., the traditional base of the chromosphere in empirical models; at $750 \mathrm{~km}$; at $1 \mathrm{Mm}$, i.e., the approximate height where the emission peaks $\mathrm{H}_{2 \mathrm{v}}$ and $\mathrm{K}_{2 \mathrm{v}}$ in the blue wings of the $\mathrm{H}$ and $\mathrm{K}$ lines of Ca II are formed; and at 1.25 Mm. The numerical model extends to a height of $1.71 \mathrm{Mm}$.

The most striking feature of the model is the omnipresence of large-amplitude temperature fluctuations. In the layers of the chromosphere, the temperature varies from below $2,000 \mathrm{~K}$ to more than 7,000 K. Even at the height of the temperature minimum in empirical models of the chromosphere, the temperature varies from less than $3,000 \mathrm{~K}$ to nearly $7,000 \mathrm{~K}$. These fluctuations are found in most of this model of the internetwork chromosphere, with regions near 2,000 K covering about half the area shown in the three cuts referring to the chromosphere. By comparison, the observed solar intensity fluctuations and the corresponding inferred brightness temperature fluctuations are much smaller. For example, the observations of the H line by Cram \& Damé (1983: hereafter CD83), which cover also the magnetic network in addition to the dark cell interior, show a fluctuation of the $\mathrm{H}_{2 \mathrm{v}}$ intensity peak by a factor of 2.2 , corresponding to the brightness temperature varying by about $1,000 \mathrm{~K}$ about the temperature of formation of the emission peak. Ninety percent of the profiles lie in the band of the profiles of CD83. At the temperature minimum $(z=500 \mathrm{~km})$, which is the formation height of the $\mathrm{H}_{1}$ minimum in the line profile, the observed temperature variation in the internetwork chromosphere plus the magnetic network is about $300 \mathrm{~K}$. Again, only $10 \%$ of the observations lie either above or below the intensity profiles of the figure.

It is evident that the temperature variations inferred from the observations are much smaller than those of the dynamical model. The question is whether the low temperature of $T \approx 2,000 \mathrm{~K}$ would be observable in the radiation emerging from the chromosphere. 

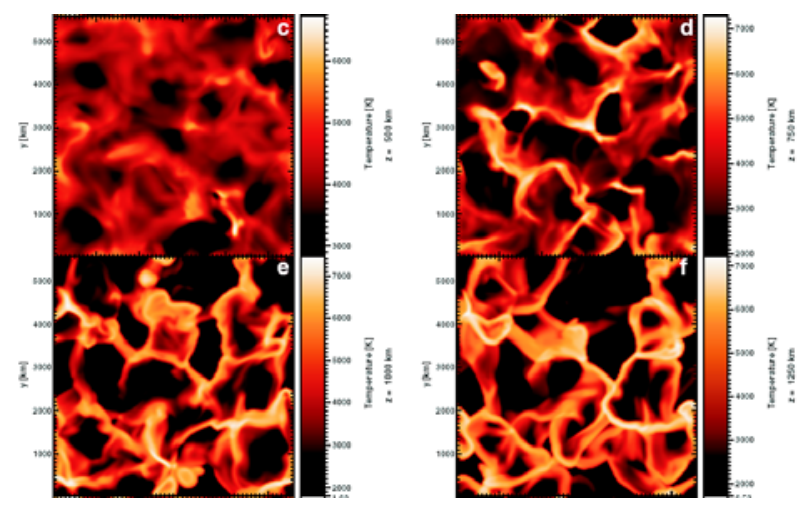

Figure 1. 3D hydrodynamical model of the internetwork chromosphere. Simultaneous cuts at $500,750,1000$, and $1250 \mathrm{~km}$ height, showing the temperature structure in horizontal layers (from Wedemeyer et al. 2004)

Consider Figure 1: The cool, dark region at the upper edge and near the right-hand border of the cuts at $750 \mathrm{~km}, 1 \mathrm{Mm}$ and $1.25 \mathrm{Mm}$ has a horizontal size of 1 to 3 arcseconds and covers a height range of at least $500 \mathrm{~km}$, and therefore about five scale heights. Figure 3 of W04 shows that such features tend to persist for at least $30 \mathrm{~s}$. Therefore, the large, dark area in this hydrodynamical model would easily be observable with ground-based instruments. And since this pattern appears to be typical for the model, the low temperature as well as the large temperature fluctuations should have been observed. The fact that they have not been noted in the emergent radiation from the chromosphere suggests that the Sun does not show such features and exhibits, instead, the much milder intensity variation of $\delta I / I_{\text {average }} \sim 2.2$ and corresponding (brightness) temperature variation of $\delta T / T_{\text {average }} \sim 15 \%$ of the $\mathrm{CD} 83$ observations at $\mathrm{H}_{2 \mathrm{v}}$ (covering the internetwork as well as the network chromosphere), or the somewhat smaller variation of the (kinetic) temperature of about $12 \%$ of the VAL models at the presumed formation height of $z \sim 1 \mathrm{Mm}$. None of these values agrees with the large temperature variation of the W04 model.

In addition to the temperature amplitude of the W04 model being larger than that inferred from $\mathrm{H}$ line observations by an order of magnitude, the dynamical time scale of the model is shorter by an order of magnitude: In the W04 model, the horizontal pattern evolves on a time scale of $20 \mathrm{~s}$ to $25 \mathrm{~s}$ whereas the observations, e.g., those of Cauzzi et al. (2007), show a time scale of $200 \mathrm{~s}$ in the atmosphere. The $200 \mathrm{~s}$ time has a natural explanation in the acoustic cutoff period in the upper photosphere.

\section{Radiative energy exchange in the wave model}

The large amplitude of the temperature fluctuations in the 3D hydrodynamical model suggests that the moderating influence of radiation in damping fluctuations is underestimated. This points to the treatment of the radiative term in the energy equation. The W04 model uses as the only significant opacity source $\mathrm{H}^{-}$bound-free and free-free transitions and represents the opacity in the form of the Rosseland mean,

$$
1 / \kappa_{\mathrm{R}}=\frac{\int 1 / \kappa_{\nu} B_{\nu} d \nu}{\int B_{\nu} d \nu} .
$$

This opacity mean measures the transparency of the medium to radiation and is weighted preferentially by frequency regions with low opacity, $\kappa_{\nu}$, and large Planck function, $B_{\nu}$. Taking the opacity at 5,000 $\AA$ as representative of the model we can estimate 
the photon mean free path from empirical models. From the VAL model A we find a mean free path of about $10^{5} \mathrm{~km}$ at $z \sim 500 \mathrm{~km}$; and throughout the chromosphere $(z>750 \mathrm{~km})$, the photon mean free path exceeds the solar diameter. Since the typical size of granules is 500 to $1000 \mathrm{~km}$, ie., smaller than the photon mean free path in the upper photosphere by two orders of magnitude, radiative energy exchange between hot and cool regions in the wave model is negligible.

The neglect of radiative energy exchange throughout the chromosphere and most of the photosphere likely accounts in large measure for the huge amplitude of temperature variation in the wave model of W04. Another peculiar feature of the model is the short time scale for the evolution of the pattern seen in the horizontal cuts in Fig. 1. Note that the typical period observed in the internetwork chromosphere is $200 \mathrm{~s}$ (see Cauzzi et al. 2007), which is readily interpreted as the acoustic cutoff period for vertical oscillations of the nonmagnetic chromosphere and which represents the response time of an atmosphere with chromospheric temperature and pressure to an impulsive wave excitation. A much shorter period has been observed by Wöger et al. (2006), with a value of $53 \mathrm{~s}$ for horizontal pattern evolution. The motions with that period have been speculated to have a chromospheric origin. But an excitation mechanism for this period, or for the 20 - $25 \mathrm{~s}$ period of the hydrodynamical model, is not apparent.

\section{Conclusion}

The energy flux of acoustic waves observed with TRACE and Hinode in the upper photosphere of the quiet Sun is insufficient to balance the radiative emission of the nonmagnetic chromosphere. Wedemeyer-Böhm et al. (2007) and Cuntz et al. (2007) have proposed that the low value of the wave flux measured by TRACE can be attributed to the low spatial resolution of the space observations, reasoning on the basis of the 3D hydrodynamical model of Wedemeyer et al. (2004). The present paper accepts the principle of spatial averaging over small-scale intensity fluctuations but finds that simplifications in the treatment of radiative energy exchange in the model magnify temperature fluctuations by an order of magnitude and prevent a quantitative estimate. Therefore, chromospheric heating by acoustic waves, or any other means, remains an unsolved and controversial problem.

\section{Acknowledgements}

I have benefited from insightful comments by R. Erdélyi. Travel support by the IAU is gratefully acknowledged.

\section{References}

Anderson, L. S. \& Athay, R. G. 1989, ApJ, 336, 1089

Bodo, G., Kalkofen, W., Massaglia, S. \& Rossi, P. 2000, A\& A, 354, 296

Carlsson, M., Hansteen, V.H., de Pontieu, B., et al. 2007, PASJ, accepted

Carlsson, M. \& Stein, R.F. 1995, ApJ, 440, L29

Cauzzi, G., Reardon, K.P., Vecchio, A., Janssen, K. \& Rimmele, T. 2007, PASP, Conf. Ser. 368, 127

Cram, L.E. \& Damé, L. 1983, ApJ, 272, 355 (CD83)

Cuntz, M., Rammacher, W. \& Musielak, Z.E. 2007, ApJ, 657, L57

Fossum, A. \& Carlsson, M. 2005a, ApJ, 625, 556

Fossum, A. \& Carlsson, M. 2005b, Nature, 435, 919

Fossum, A. \& Carlsson, M. 2006, ApJ, 646, 579

Kalkofen, W. 2007, ApJ, 671, in press 
Lites, B.W., Rutten, R.J. \& Kalkofen, W. 1993, ApJ, 414, 345

Liu, S.-Y. 1974, ApJ, 189, 359

Mendoza-Briceño, C. A. \& Erdélyi, R. 2006, ApJ, 648, 722

Vernazza, J.E., Avrett, E.H. \& Loeser, R. 1981, ApJS, 45, 635 (VAL)

Wedemeyer, S., Freytag, B., Steffen, M., Ludwig, H.-G., \& Holweger, H. 2004, A\& A, 414, 1121 (W04)

Wedemeyer-Böhm, S., Steiner, O., Bruls, J., \& Rammacher, W. 2007, PASP Conf. Ser. 368, 93

Wöger, F., Wedemeyer-Böhm, S., Schmidt, W. \& von der Lühe, O. 2006, A $\& A$ 459, L9 